\title{
Acute Stroke Thrombolysis in End-Stage Renal Disease: A National Survey of Nephrologist Opinion
}

\author{
Albert Power $^{\mathrm{a}}$ Damian Fogarty ${ }^{\mathrm{c}}$ David C. Wheeler ${ }^{\mathrm{b}}$ \\ ${ }^{a}$ Haemodialysis Research Group, Imperial College Kidney and Transplant Centre, Imperial College Healthcare NHS \\ Trust, Hammersmith Hospital, and b UCL Centre for Nephrology, UCL Medical School, Royal Free Campus, London, and \\ 'UK Renal Registry, Southmead Hospital, Bristol, UK
}

\section{Key Words}

End-stage renal disease $\cdot$ Stroke $\cdot$ Thrombolysis · Dialysis

\begin{abstract}
Background/Aims: Systemic thrombolysis for acute ischaemic stroke is the standard of care in the UK. In the absence of trial data on the safety and efficacy of this treatment in patients with end-stage renal disease, we captured the views of UK nephrologists to highlight health care policy and research objectives. Methods: Consultant nephrologists participated in an internet-based questionnaire. Respondents were asked about their involvement in thrombolysis decisions, safety concerns in dialysis patients, views on stroke rehabilitation and opinions on antiplatelet and warfarin use for stroke prevention. Results: 122/433 (28\%) clinicians responded. $75 \%$ wanted involvement in thrombolysis decisions although just $10 \%$ gave input in practice. $64 \%$ expressed a high degree of concern $(\geq 7 / 10)$ regarding intracranial bleeding risk in haemodialysis (HD). Overall risks of intra- and extracranial bleeding were rated lower in peritoneal dialysis (PD; $p<0.001$ ). $85 \%$ felt the HD schedule impacted negatively on rehabilitation, whereas $63 \%$ felt this was the case in the context of PD $(p=0.001)$. More clinicians favoured warfarin for stroke prevention in PD patients with
\end{abstract}

\begin{tabular}{|c|c|}
\hline KARGER & $\begin{array}{l}\text { (c) } 2013 \text { S. Karger AG, Basel } \\
1660-2110 / 13 / 1244-0167 \$ 38.00 / 0\end{array}$ \\
\hline $\begin{array}{l}\text { E-Mail karger@karger.com } \\
\text { www.karger.com/nec }\end{array}$ & $\begin{array}{l}\text { This is an Open Access article licensed under the terms of the } \\
\text { Creative Commons Attribution-NonCommercial } 3.0 \text { Un- } \\
\text { ported license (CC BY-NC) (www.karger.com/OA-license), } \\
\text { applicable to the online version of the article only. Distribu- } \\
\text { tion permitted for non-commercial purposes only. }\end{array}$ \\
\hline
\end{tabular}

atrial fibrillation in comparison with HD patients (79 vs. $66 \%$, $p=0.04)$. Conclusion: The majority of nephrologists want involvement in thrombolysis decisions relevant to their patients. Concerns about bleeding risks with thrombolysis are high and we identify a vital need to improve access to stroke rehabilitation in the UK, especially in HD patients.

(c) 2013 S. Karger AG, Basel

\section{Introduction}

Stroke remains a major cause of death and disability with an incidence in dialysis patients 10-15 times higher than the general population, and it is associated with a worse prognosis $[1,2]$. This predilection to stroke is attributed to a higher prevalence of conventional vascular risk factors and dialysis-specific causes: accelerated calcific arteriosclerosis, vascular access and the use of anticoagulants to maintain flow in the extracorporeal circuit.

Acute ischaemic stroke accounts for approximately $70-80 \%$ of strokes in dialysis patients. Urgent recanalization can be achieved using mechanical embolectomy or systemic thrombolysis [3]. When delivered within $6 \mathrm{~h}$ of symptom onset in the general population, alteplase results in a significant improvement in neurodeficit and

Dr. Albert Power, MD

Imperial College Renal and Transplant Centre

Hammersmith Hospital

Du Cane Road, London W12 0HS (UK)

E-Mail a.power08@imperial.ac.uk 
functional disability compared to placebo [4], with a low but appreciable risk of haemorrhagic transformation which increases further when given $>6 \mathrm{~h}$ from symptom onset [5].

To date the risks and benefits of stroke thrombolysis in dialysis patients remain undefined. Renal impairment is not a licensed contra-indication to alteplase with no trials to date exploring the effect of kidney function on outcome. Uraemia confers a bleeding diathesis which is particularly marked in patients with end-stage renal disease (ESRD) [6]. This is further accentuated with concomitant antiplatelet use, regular exposure to heparins during haemodialysis (HD) and the effects of hypertension and hypervolaemia. It can therefore be hypothesised that the haemorrhagic risk of thrombolysis may outweigh any benefits. This concerned the majority of stroke experts polled in the only previous survey conducted on this topic [7].

The opinions and experience of nephrologists or kidney patients have never been solicited to date. Furthermore no study has explored the current clinical practice in acute stroke management for patients on peritoneal dialysis (PD) as well as those receiving HD. We therefore sought to comprehensively ascertain the opinions of nephrologists on acute stroke management and thrombolysis in the dialysis cohorts under their care.

\section{Methods}

Nephrology services across the UK are delivered by large renal units, some with and some without transplantation programmes. At the time of this survey, 72 renal units (23 with transplantation) covered the population of the UK staffed by approximately 460 consultant renal physicians. We contacted all consultant nephrologists within the UK by e-mail to participate anonymously in an internet-based survey. A second invitation was sent to clinicians who had not responded to the initial e-mail within 2 weeks, and all responses were evaluated 1 month after the initial request.

We aimed to capture opinions on a number of facets of care around stroke thrombolysis including the role of nephrologists in the decision-making process, areas of concern regarding acute stroke care and thrombolysis, modifications to the dialysis prescription and what adjuvant pharmacotherapy clinicians supported (for questionnaire structure, see online suppl. material; for all online suppl. material, see www.karger.com/doi/10.1159/000357155).

In the domains where some responses were incomplete, the denominator is given in the text. Descriptive statistics were expressed as the mean \pm standard deviation and the median with interquartile range as appropriate. Continuous and categorical variables were compared using Student's t test and the $\chi^{2}$ or MannWhitney U test, respectively, as appropriate. All analyses were performed using STATA 12.1 (www.stata.com). Statistical significance was defined by $\mathrm{p}<0.05$.

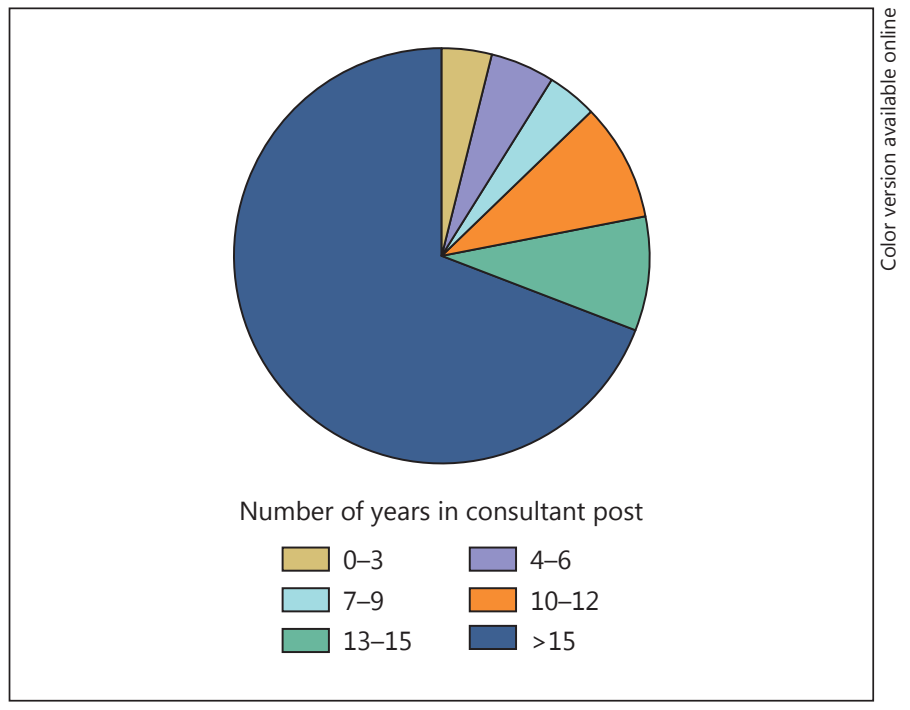

Fig. 1. Clinical experience of survey respondents.

\section{Results}

Of the 433 clinicians who were invited, 122 (28\%) from 55 centres responded. $69 \%$ of the respondents had $>15$ years' practice at the consultant level (fig. 1) with $97 \%$ providing inpatient cover. The median size of the dialysis programme in each centre was 450 patients $(73-1,450)$ with PD patients accounting for $16.0 \pm 9.6 \%$ of the total cohort size on average.

$23 \%$ of the respondents had dialysis patients in their centres who received stroke thrombolysis. The overall centre experience varied from 0 to 10 patients reflecting the dialysis programme size (fig. 2). Only $10 \%$ were actually involved in thrombolysis decisions, and yet $75 \%$ reported a wish for some involvement in the process, with $82 \%$ wanting contact immediately prior to thrombolysis. The reasons cited included concerns about the risks of thrombolysis weighed against uncertainties about the benefits of this treatment in a particularly comorbid subgroup.

\section{Key Concerns about Stroke Thrombolysis in Dialysis \\ Patients}

Concerns regarding the risk of bleeding with thrombolytic therapy predominated with a significantly higher risk perceived in $\mathrm{HD}$ patients as compared to those on $\mathrm{PD}$ (score of 7/10 vs. 6/10) applicable to intracranial and extracranial bleeding events (table 1). A high bleeding risk with thrombolysis (rated $\geq 7 / 10$ ) was attributed to HD pa- 


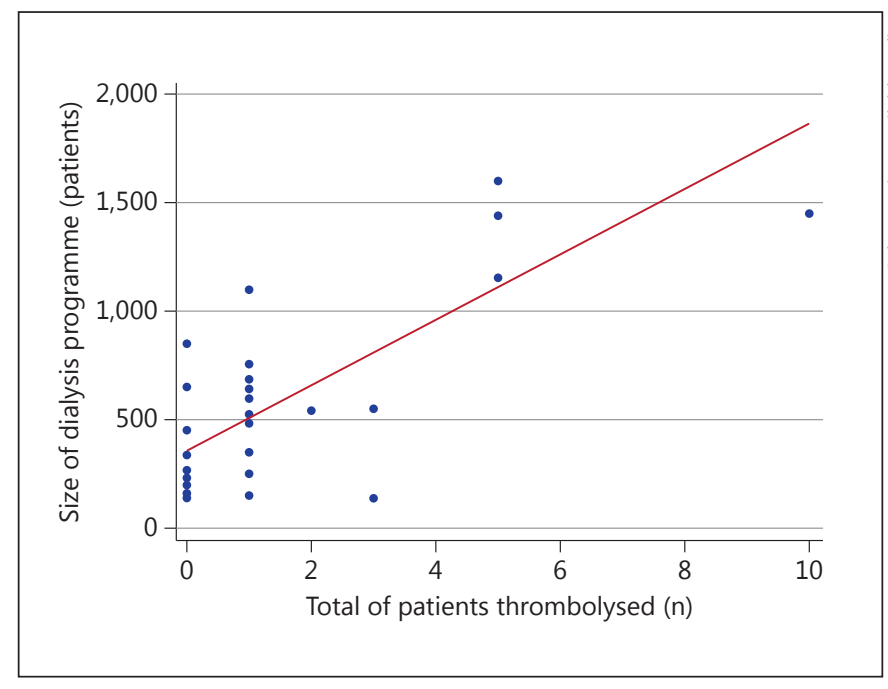

Fig. 2. Experience of stroke thrombolysis in ESRD patients according to the size of dialysis programme.

Table 1. Extent of clinical concern expressed by nephrologists regarding the use of thrombolytic therapy for acute stroke according to dialysis modality

\begin{tabular}{llll}
\hline & \multicolumn{2}{l}{ Degree of clinical concern } & $\mathrm{p}$ \\
\cline { 2 - 3 } & value \\
& HD & PD & \\
\hline Risk of intracerebral bleeding & $7(5-8)$ & $6(5-7)$ & 0.01 \\
Risk of extracerebral bleeding & $6(4-8)$ & $5(4-7)$ & 0.03 \\
Cardiovascular stability & $4(3-5)$ & $4(2-5)$ & 0.21 \\
Efficacy & $5(3-6)$ & $5(4-6)$ & 0.91 \\
Evidence base for use & $6(3-7)$ & $6(3-7)$ & 0.61 \\
Logistics of dialysis delivery & $5(3-6)$ & $3(2-5)$ & 0.001 \\
Drug dosing by stroke teams & $5(3-7)$ & $5(3-7)$ & 0.26 \\
Other & & & \\
\hline
\end{tabular}

Concern: 1 = negligible, $10=$ overwhelming. Median values are displayed with the interquartile ranges in parentheses.

tients by $78 / 122$ (64\%) clinicians compared with $60 / 122$ $(49 \%)$ for PD patients $(\mathrm{p}=0.02)$.

Clinicians reported a moderate degree of concern regarding the impact of dialysis on cardiovascular stability around the time of thrombolysis. There was no significant difference according to the dialysis modality used (table 1). Similarly a moderate degree of concern was expressed regarding the perceived efficacy of this treatment as well as issues relating to drug dosing of dialysis patients by stroke teams rather than nephrologists (table 1) with no significant differences between HD and PD patients. There was however an increased level of concern regarding the logistics of delivering HD compared to PD following thrombolysis $(\mathrm{p}=0.001)$.

On average a moderate degree of concern (6/10) was raised regarding the available evidence base supporting the use of thrombolytic therapy for acute stroke in dialysis patients which did not vary according to dialysis modality.

\section{Modification of the Dialysis Prescription in Acute Stroke Patients}

$76 \%$ of the clinicians would modify the HD prescription including some of the following:

- Avoiding HD unless imperative for the first 24-48 h

- Minimisation/avoidance of heparins

- Reduction in dialytic efficiency (lower blood pump speeds, reductions in dialyser surface area, shorter dialysis times)

- Daily dialysis or haemofiltration

- Limiting ultrafiltration rates

In contrast, 20/108 (19\%) clinicians would modify the PD prescription. Responses were more general in nature incorporating reductions in ultrafiltration volume and maintaining haemodynamic stability. A quarter of respondents who provided further detail either deferred any changes to their PD colleagues or expressed a lack of expertise in this area.

\section{Impact of Dialysis Therapy on Rehabilitation after Acute Stroke}

The majority of respondents felt that the dialysis schedule impacted negatively on rehabilitation. This was more marked for HD patients (85\%) compared to those on PD $(64 \%, \mathrm{p}=0.001)$.

The adverse effect of HD on rehabilitation was attributed to issues such as:

- Treatment time precluding access to rehabilitative services such as physiotherapy

- Transport to and from the dialysis unit

- Dialysis 'wash-out' (i.e. time to recovery)

- Dialysis mandating an inpatient stay on a nephrology ward as opposed to a dedicated stroke unit

- Comorbidities limiting the scope for functional improvement

\section{Antiplatelet/Anticoagulant Therapies after Stroke}

Over $75 \%$ of clinicians would initiate or augment antiplatelet therapies within $48 \mathrm{~h}$ of thrombolysis (table 2). More clinicians would use warfarin for stroke prevention 
Table 2. Nephrologist opinion on the timing of initiation or augmentation of antiplatelet therapy after acute stroke according to dialysis modality

\begin{tabular}{lllllllll}
\hline Dialysis modality & \multicolumn{2}{l}{ Time from thrombolysis } \\
\cline { 2 - 8 } & within $12 \mathrm{~h}$ & $12-24 \mathrm{~h}$ & $24-48 \mathrm{~h}$ & 3 days & 4 days & 1 week & 2 weeks & $>2$ weeks \\
\hline HD $(\mathrm{n}=121)$ & $24(20)$ & $45(37)$ & $24(20)$ & $4(3)$ & 0 & $4(3)$ & $2(2)$ & $2(2)$ \\
$\mathrm{PD}(\mathrm{n}=111)$ & $30(27)$ & $40(36)$ & $24(22)$ & $2(2)$ & 0 & $5(5)$ & $3(3)$ & $1(1)$ \\
\hline
\end{tabular}

Data are given as number of responses with percentages in parentheses.

in PD patients with atrial fibrillation than in $\mathrm{HD}$ (79 vs. $66 \%, p=0.04)$. This mainly related to bleeding risks associated with warfarin, and a number of clinicians favoured risk stratification using clinical scores (e.g. HASBLED, CHA2DS2-VASc) with individualised treatment decisions based on comorbidity.

\section{Concluding Comments}

Overall $76 \%$ of the respondents expressed a wish to participate in national studies examining clinical outcomes following stroke thrombolysis. There was no suggestion of conducting a stroke trial comparing thrombolysis to non-thrombolytic care. A number of respondents identified research areas of priority such as trials of warfarin versus antiplatelet therapy alone for stroke prevention in ESRD patients with atrial fibrillation, and on intracerebral haemorrhage in dialysis patients.

\section{Discussion}

Stroke thrombolysis in ESRD is dominated by concerns regarding bleeding risks and subsequent access to effective rehabilitation. Concerns regarding bleeding resonate with those of stroke physicians and remain an enduring treatment dilemma [7]. Higher bleeding rates with declining renal function in primary thrombolysis for myocardial infarction have been reported in a Swedish registry study but it is unclear whether this is an effect of treatment choice or renal dysfunction per se [8]. The absence of trial data on the safety of thrombolysis in ESRD was reinforced as a significant issue in our study. Previously $78 \%$ of stroke experts favoured intraarterial approaches rather than systemic thrombolysis in HD [7].

We found a moderate degree of concern regarding the efficacy of thrombolysis in dialysis patients relating to a subjective appraisal of comorbid burden and baseline functional level rather than issues with the lytic efficacy of alteplase in ESRD. Patients with ESRD form compact blood clots in vitro that are more resistant to lysis [9], but it is unclear whether this results in clinically significant effects in stroke thrombolysis. Furthermore dialysis patients have a high prevalence of clinically silent structural brain disease (e.g. infarcts, microbleeds) which may attenuate the thrombolytic benefit [10-12], in keeping with data suggesting that impaired kidney function is an independent risk factor for suboptimal post-thrombolysis outcomes [13-15].

Dialysis requires specialist nursing input, dedicated equipment and, in the case of in-centre $\mathrm{HD}$, a dedicated clinical environment. Stroke thrombolysis is delivered in hyperacute stroke units in the UK under the direction of specialist teams. As a result acute stroke care and rehabilitation can be geographically separate from dialysis facilities. This dichotomy of care at a critical point in the patient pathway was highlighted as a major barrier to rehabilitation, especially for HD patients who require a higher level of technological and nursing support compared to those on PD. To date there are no published studies on rehabilitation outcomes following stroke in ESRD patients. Minimizing the time to recovery from $\mathrm{HD}$ therapy may improve engagement with rehabilitation services [16], and co-ordinating dialysis treatments around rehabilitation may represent a simple, high-impact intervention. The integration of care remains challenging, and to date only 1 renal centre in the UK has a stroke unit embedded in its nephrology wards.

We found a striking disparity in the proportion of clinicians who tailor the dialysis prescription after acute stroke according to modality. Published recommendations for HD patients after acute brain injury derive in part from data obtained in traumatic brain injury which may not be applicable to stroke [17]. Similar recommen- 
dations in PD are lacking, and there are no studies to date examining the acute impact of HD or PD on the brain of acute stroke patients.

Despite appreciating platelet dysfunction in ESRD most UK nephrologists initiated or incremented antiplatelet therapies early after thrombolysis. The role of warfarin for stroke thromboprophylaxis in dialysis patients with atrial fibrillation remains contentious. Observational data in HD has linked warfarin use with a higher stroke risk in some studies [18], whereas a recent study suggested the opposite [19]. Nonetheless, warfarin conferred a higher bleeding risk in that series in keeping with prior data $[20,21]$. Furthermore data suggests that warfarin can increase the cardiovascular risk directly by accelerated vascular calcification [22].

Our study constitutes the first appraisal of issues around stroke thrombolysis in dialysis reflecting topical concerns, therapeutic barriers and practical approaches to the management of patients. Our study is limited by a relatively low response rate despite parity with survey response rates in the literature [23]. Therefore our data may be prone to occult bias. In addition nephrologists with longer tenure in post were likely overrepresented in this study given that around $36 \%$ of the nephrologists in the UK have $>15$ years of consultant experience compared to $69 \%$ in this survey $(\mathrm{p}<0.001)$. As a result, our findings could be vulnerable to confounding by deriving from a distinct, self-selected group of clinicians who nonetheless bring a wealth of clinical experience and are more likely to influence other professional opinions. A clustering of responses particularly from large urban centres (e.g. London and Glasgow) may have influenced our results further and curb the generalizability of our findings. Finally this study represents an assessment of specialist opinion which is not a substitute for clinical trial data.

There is enduring concern regarding systemic thrombolysis in dialysis cohorts that appear prejudiced with respect to their rehabilitation. We believe that large collaborative studies are needed to determine the safety of this therapy in dialysis patients and the optimal care pathways to ensure the best post-stroke outcomes. The efficacy and safety of warfarin in stroke thromboprophylaxis remains contentious, especially in HD patients, and trials are required to determine the risks and benefits of this agent in ESRD.

\section{Disclosure Statement}

The authors have no financial or non-financial conflicts of interest to declare.

\section{References}

$>1$ Power A, Chan K, Singh SK, Taube D, Duncan N: Appraising stroke risk in maintenance hemodialysis patients: a large single-center cohort study. Am J Kidney Dis 2012;59:249257.

-2 Seliger SL, Gillen DL, Tirschwell D, Wasse H, Kestenbaum BR, Stehman-Breen CO: Risk factors for incident stroke among patients with end-stage renal disease. J Am Soc Nephrol 2003;14:2623-2631.

$>3$ Alexandrov AV: Current and future recanalization strategies for acute ischemic stroke. J Intern Med 2010;267:209-219.

4 IST-3 Collaborative Group, Sandercock P, Wardlaw JM, Lindley RI, Dennis M, Cohen G, et al: The benefits and harms of intravenous thrombolysis with recombinant tissue plasminogen activator within $6 \mathrm{~h}$ of acute ischaemic stroke [the third international stroke trial (IST-3)]: a randomised controlled trial. Lancet 2012;379:2352-2363.

$>5$ Wahlgren N, Ahmed N, Eriksson N, Aichner F, Bluhmki E, Davalos A, et al: Multivariable analysis of outcome predictors and adjustment of main outcome results to baseline data profile in randomized controlled trials: Safe
Implementation of Thrombolysis in StrokeMonitoring Study (SITS-MOST). Stroke 2008; 39:3316-3322.

-6 Galbusera M, Remuzzi G, Boccardo P: Treatment of bleeding in dialysis patients. Semin Dial 2009;22:279-286.

$>7$ Palacio S, Gonzales NR, Sangha NS, Birnbaum LA, Hart RG: Thrombolysis for acute stroke in hemodialysis: international survey of expert opinion. Clin J Am Soc Nephrol 2011;6:1089-1093.

8 Szummer K, Lundman P, Jacobson SH, Schon $\mathrm{S}$, Lindback J, Stenestrand U, et al: Relation between renal function, presentation, use of therapies and in-hospital complications in acute coronary syndrome: data from the SWEDEHEART register. J Intern Med 2010; 268:40-49.

-9 Sjoland JA, Sidelmann JJ, Brabrand M, Pedersen RS, Pedersen JH, Esbensen K, et al: Fibrin clot structure in patients with end-stage renal disease. Thromb Haemost 2007;98:339-345.

10 Drew DA, Bhadelia R, Tighiouart H, Novak V, Scott TM, Lou KV, et al: Anatomic brain disease in hemodialysis patients: a cross-sectional study. Am J Kidney Dis 2013;61:271-278.
11 Naganuma T, Takemoto Y, Yamasaki T, Shima H, Shoji T, Ishimura E, et al: Factors associated with silent cerebral microbleeds in hemodialysis patients. Clin Nephrol 2011;75: 346-355.

12 Watanabe A: Cerebral microbleeds and intracerebral hemorrhages in patients on maintenance hemodialysis. J Stroke Cerebrovasc Dis 2007;16:30-33.

13 Lyrer PA, Fluri F, Gisler D, Papa S, Hatz F, Engelter ST: Renal function and outcome among stroke patients treated with IV thrombolysis. Neurology 2008;71:1548-1550.

14 Naganuma M, Koga M, Shiokawa Y, Nakagawara J, Furui E, Kimura K, et al: Reduced estimated glomerular filtration rate is associated with stroke outcome after intravenous rtPA: the Stroke Acute Management with Urgent Risk-Factor Assessment and Improvement (SAMURAI) rt-PA registry. Cerebrovasc Dis 2011;31:123-129.

- 15 Power A, Epstein D, Cohen D, Bathula R, Devine J, Kar A, et al: Renal impairment reduces the efficacy of thrombolytic therapy in acute ischemic stroke. Cerebrovasc Dis 2013; 35:45-52. 
16 Jaber BL, Lee Y, Collins AJ, Hull AR, Kraus MA, McCarthy J, et al: Effect of daily hemodialysis on depressive symptoms and postdialysis recovery time: interim report from the FREEDOM (Following Rehabilitation, Economics and Everyday-Dialysis Outcome Measurements) Study. Am J Kidney Dis 2010; 56:531-539.

$\checkmark 17$ Davenport A: Practical guidance for dialyzing a hemodialysis patient following acute brain injury. Hemodial Int 2008;12:307-312.

18 Chan KE, Lazarus JM, Thadhani R, Hakim RM: Warfarin use associates with increased risk for stroke in hemodialysis patients with atrial fibrillation. J Am Soc Nephrol 2009;20: 2223-2233.
19 Olesen JB, Lip GY, Kamper AL, Hommel K, Kober L, Lane DA, et al: Stroke and bleeding in atrial fibrillation with chronic kidney disease. N Engl J Med 2012;367:625-635.

20 Winkelmayer WC, Liu J, Setoguchi S, Choudhry NK: Effectiveness and safety of warfarin initiation in older hemodialysis patients with incident atrial fibrillation. Clin J Am Soc Nephrol 2011;6:2662-2668.
21 Wizemann V, Tong L, Satayathum S, Disney A, Akiba T, Fissell RB, et al: Atrial fibrillation in hemodialysis patients: clinical features and associations with anticoagulant therapy. Kidney Int 2010;77:1098-1106.

22 Kruger T, Oelenberg S, Kaesler N, Schurgers LJ, van de Sandt AM, Boor P, et al: Warfarin induces cardiovascular damage in mice. Arterioscler Thromb Vasc Biol 2013;33:26182624.

23 Kaplowitz MD, Hadlock TD, Levine R: A comparison of web and mail survey response rates. Public Opin Q 2004;68:94-101. 